\title{
Spastic Paraplegia Accompanied by Extrapyramidal Sign and Frontal Cognitive Dysfunction
}

\author{
Ryo Sasaki ${ }^{1}$, Yasuyuki Ohta ${ }^{1}$, Kota Sato ${ }^{1}$, Koh Tadokoro ${ }^{1}$, Yoshiaki Takahashi ${ }^{1}$, \\ Jingwei Shang ${ }^{1}$, Mami Takemoto ${ }^{1}$, Nozomi Hishikawa ${ }^{1}$, Toru Yamashita ${ }^{1}$, Hiroyuki Ishiura ${ }^{2}$, \\ Shoji Tsuji ${ }^{3,4}$ and Koji Abe ${ }^{1}$
}

\begin{abstract}
:
A complicated form of spastic paraplegia is a neurodegenerative disorder presenting as progressive spasticity in the bilateral lower limbs accompanied by some clinical features. The present case showed spastic paralysis and hyperreflexia in all extremities as well as lead pipe rigidity in the neck and bilateral upper extremities $(\mathrm{R}<\mathrm{L})$, decreased scores on frontal cognitive tests, a decreased accumulation of the right dorsal putamen on a DAT scan, and hypoperfusion of the bilateral frontal lobes on ${ }^{99 \mathrm{~m}} \mathrm{Tc}$-ECD single photon emission computed tomography (SPECT). The present case provides a new spectrum of spastic paraplegia based on the evidence of clinical scores and the findings of brain functional imaging.
\end{abstract}

Key words: spastic paraplegia, extrapyramidal sign, frontal cognitive dysfunction

(Intern Med 58: 3163-3165, 2019)

(DOI: 10.2169/internalmedicine.2765-19)

\section{Introduction}

Spastic paraplegia is a heterogeneous group of inherited neurological disorders in which the main clinical feature is progressive lower limb spasticity. A complicated form of spastic paraplegia is accompanied by additional neurological features such as mental retardation, cognitive impairment, ataxia, neuropathy or other symptoms (1), but a few cases have shown extrapyramidal signs $(2,3)$ without any general cognitive impairment (4) or frontal lobe dysfunction (5). We herein report the known first case of spastic paraplegia accompanied by both an extrapyramidal sign and frontal cognitive dysfunction which was evaluated by cognitive tests and the findings of brain functional imaging.

\section{Case Report}

A 6-year-old boy was not good at running fast, but he had no problem with walking. However, symptoms associ- ated with spasticity gradually developed and he could not play any sports well in his schooldays or even in adulthood. $\mathrm{He}$ developed a gait disturbance at 60 years of age, difficulty in climbing stairs at 63 years of age, and a tendency to fall easily while walking at 65 years of age. He was subsequently admitted to our hospital at 67 years of age. He had been diagnosed to have bilateral otitis media at 9 years of age, but had no particular history of neurological disease, mental abnormalities, behavioral disorder nor any family history of intermarriage, although his father's family and mother's family were from Osaka and Hikone, which are both in the Kansai area of Japan.

He was $164.0 \mathrm{~cm}$ tall and weighed $51.8 \mathrm{~kg}$. A neurological examination showed the presence of mild bradykinesia, a mild lower gaze limitation, severe hearing loss in his bilateral ears (right $>$ left), and mild lingual dysarthria. He also presented with mild muscle weakness in his neck and bilateral lower extremities (Manual muscle testing; neck and bilateral L/E 4/5), severe spasticity in all extremities (A/E), spastic gait, bilateral pes cavus, mild lead pipe rigidity in his

\footnotetext{
${ }^{1}$ Department of Neurology, Graduate School of Medicine, Dentistry and Pharmaceutical Sciences, Okayama University, Japan, ${ }^{2}$ Department of Neurology, The University of Tokyo Hospital, Japan, ${ }^{3}$ Department of Molecular Neurology, The University of Tokyo Hospital, Japan and ${ }^{4}$ Institute of Medical Genomics, International University of Health and Welfare, Japan

Received: February 1, 2019; Accepted: May 7, 2019; Advance Publication by J-STAGE: July 10, 2019

Correspondence to Dr. Koji Abe, ryosasaki@okayama-u.ac.jp
} 

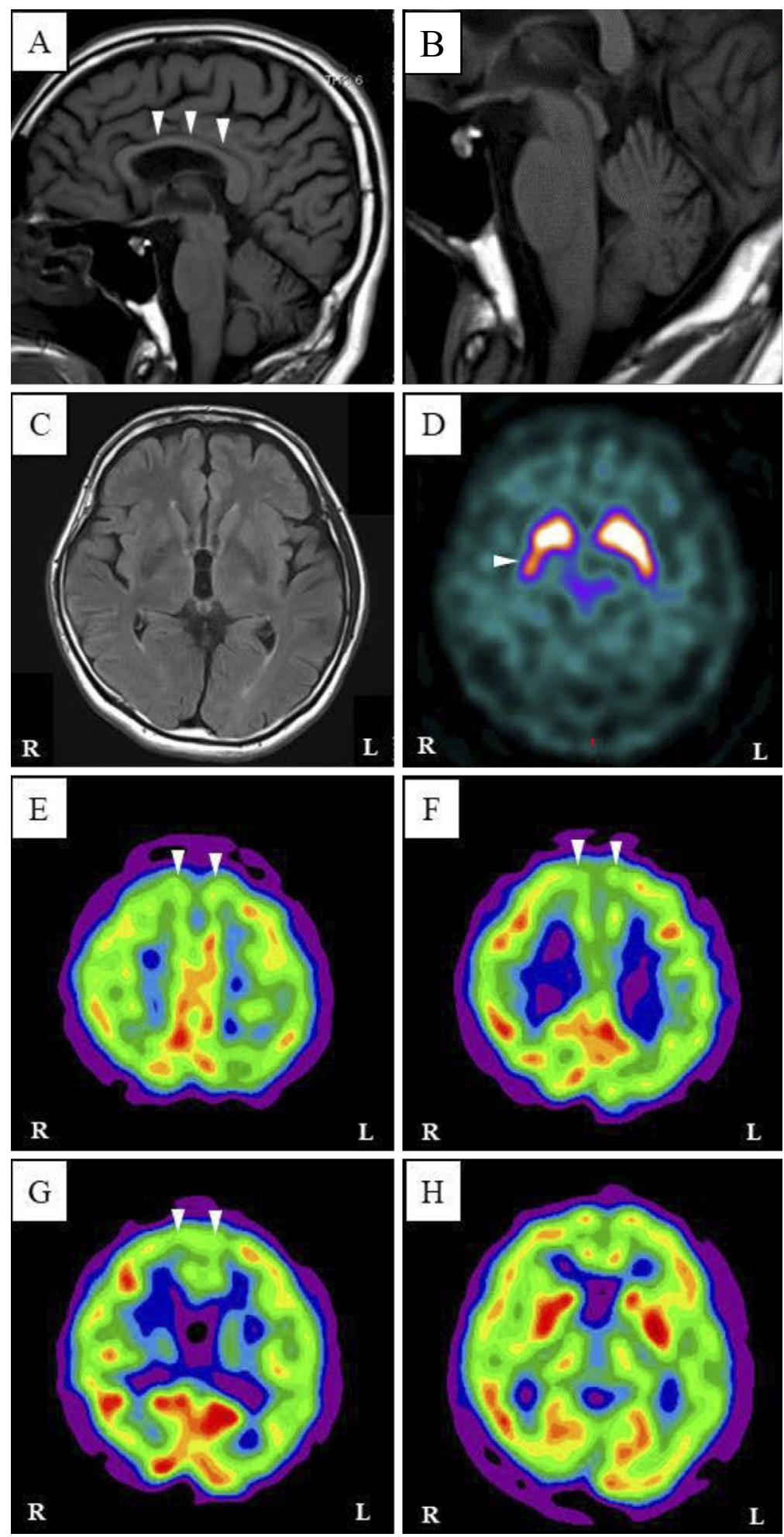

Figure. (A-C) Brain MRI showing a thin corpus callosum (A, arrowheads), but no significant lesions in the brainstem or bilateral basal ganglia (B and C). (D) A DAT scan showing a decreased accumulation in the right dorsal putamen (arrowhead). (E-H) ${ }^{99 \mathrm{~m}}$ Tc-ECD SPECT showing hypoperfusion in the bilateral frontal lobes (arrowheads).

neck and bilateral elbows and wrists and retropulsion. General cognitive tests were otherwise normal with a mini mental state examination (MMSE) score of 28/30, and a Hasegawa dementia rating scale-revised (HDS-R) score of 29/30. However, his frontal cognitive function had decreased with a frontal assessment battery (FAB) score of 11/18, and a Montreal cognitive assessment (MoCA) score of 20/30.
Serum analyses showed a normal creatine phosphokinase (CPK) level (210 U/L, normal 59-248 U/L) and negative antibodies to human T-lymphotropic virus 1 (HTLV-1) and human immunodeficiency virus (HIV) as well as normal findings for a spinal fluid analysis. A nerve conduction study showed no abnormalities in his tibial nerves.

Brain magnetic resonance imaging (MRI) showed a 
slightly thin corpus callosum (Figure A, arrowheads), but no significant lesions in the mesencephalic tegmentum (Figure B), bilateral basal ganglia, white matter, or the pyramidal tract (Figure C). ${ }^{123}$ I-Ioflupane single-photon emission computed tomography ( ${ }^{123}$ I-Ioflupane SPECT: DAT scan) showed a slightly decreased accumulation in the right dorsal putamen (SBR; right 6.15, left 7.02, average of the same age data 7.45, Z-score; right -0.96, left -0.32) (Figure D, arrowhead). ${ }^{99 \mathrm{~m}}$ Tc-ethyl cysteinate dimer $\left({ }^{99 \mathrm{~m}} \mathrm{Tc}-\mathrm{ECD}\right) \mathrm{SPECT}$ showed hypoperfusion in the bilateral frontal lobes (Figure E-H, arrowheads). We carried out the whole exome sequencing of genomic DNA (Tokyo University, Japan), but no pathological variants in the coding exons were observed.

He was therefore diagnosed to have a complicated form of spastic paraplegia accompanied by an extrapyramidal sign and frontal cognitive dysfunction. He was treated by baclofen (15 mg/day, orally) and L-dopa (increased to $600 \mathrm{mg}$ / day), but his symptoms did not improve significantly. He has been followed by a neurologist at a nearby clinic.

\section{Discussion}

Spastic paraplegia is a heterogeneous neurodegenerative disorder presenting with progressive spasticity and muscle weakness in bilateral L/E. Spastic paraplegia is clinically divided into two subtypes based on the absence or presence of combined clinical features such as mental retardation, cognitive impairment, ataxia, neuropathy, extrapyramidal sign, or other symptoms (1). Although a few cases of SPG11 presented as early-onset parkinsonism $(6,7)$ and other cases of spastic paraplegia were accompanied by a thin corpus callosum plus parkinsonism (2), parkinsonism is rarely found in complicated spastic paraplegia. Similar to the present case (Figure D, arrowhead), a previous case of spastic paraplegia case showed a decreased accumulation of DAT with parkinsonism (8). The present case showed severe spasticity in all extremities (A/E) plus mild bradykinesia, mild lead pipe rigidity in his neck and bilateral elbows and wrists, retropulsion, and a decreased accumulation of DAT (Figure D, arrowhead), thus suggesting the presence of spastic paraplegia accompanied by parkinsonism.

In contrast to well preserved general cognitive functions, the frontal lobe function had evidently decreased in the present case to $11 / 18$ of FAB and 20/30 of MoCA, concomitant with hypoperfusion of bilateral frontal lobes (Figure E-H). There are some types of hereditary spastic paraplegia with a thin corpus callosum such as SPG 11, 15, and $21(9,10)$, accompanied by parkinsonism, cognitive dysfunction, or frontal lobe dysfunction. However, no previously reported SPG case has ever been reported to show all of these symptoms. In addition, the whole-exon sequence method is associated with some limitations due to the fact that the intron has not yet been comprehensively analyzed, and it also re- mains unclear as to whether or not some new variants are pathogenic. In the future, further genetic analyses are needed to clarify the present case as a possible subtype of complicated spastic paraplegia.

This is the first case report of spastic paraplegia accompanied by both an extrapyramidal sign and frontal cognitive dysfunction, and which was diagnosed based on the findings of cognitive tests and brain functional imaging. The present case provides a new spectrum of spastic paraplegia based on the findings of clinical scores, a DAT scan and ECDSPECT.

\section{The authors state that they have no Conflict of Interest (COI).}

\section{Financial Support}

This work was partly supported by a Grant-in-Aid for Scientific Research (B) 17H0419619, (C) 15K0931607, 17H0419619 and $17 \mathrm{~K} 1082709$, and by Grants-in-Aid from the Research Committees (Kaji R, Toba K, and Tsuji S) from the Japan Agency for Medical Research and Development (AMED) 7211700176, 7211700180 and 7211700095 .

\section{References}

1. Harding AE. Classification of the hereditary ataxias and paraplegias. Lancet 1: 1151-1155, 1983.

2. Kang SY, Lee MH, Lee SK, Sohn YH. Levodopa-responsive parkinsonism in hereditary spastic paraplegia with thin corpus callosum. Parkinsonism Relat Disord 10: 425-427, 2004.

3. Guidubaldi A, Piano C, Santorelli FM, et al. Novel mutations in SPG11 cause hereditary spastic paraplegia associated with earlyonset levodopa-responsive Parkinsonism. Mov Disord 26: 553-556, 2011.

4. Faber I, Branco LMT, Franca Junior MC. Cognitive dysfunction in hereditary spastic paraplegias and other motor neuron disorders. Dement Neuropsychol 10: 276-279, 2016.

5. Yanase D, Komai K, Hamaguchi T, et al. Hereditary spastic paraplegia with frontal lobe dysfunction: a clinicopathologic study. Neurology 63: 2149-2152, 2004.

6. Vanderver A, Tonduti D, Auerbach S, et al. Neurotransmitter abnormalities and response to supplementation in SPG11. Mol Genet Metab 107: 229-233, 2012.

7. Anheim M, Lagier-Tourenne C, Stevanin G, et al. SPG11 spastic paraplegia. A new cause of juvenile parkinsonism. J Neurol 256: 104-108, 2009

8. Kim JS, Kim JM, Kim YK, Kim SE, Yun JY, Jeon BS. Striatal dopaminergic functioning in patients with sporadic and hereditary spastic paraplegias with parkinsonism.. J Korean Med Sci 28: 1661-1666, 2013.

9. Renvoise B, Chang J, Singh R, et al. Lysosomal abnormalities in hereditary spastic paraplegia types SPG15 and SPG11. Ann Clin Transl Neurol 1: 379-389, 2014.

10. Simpson MA, Cross H, Proukakis C, et al. Maspardin is mutated in mast syndrome, a complicated form of hereditary spastic paraplegia associated with dementia. Am J Hum Genet 73: 1147-1156, 2003.

The Internal Medicine is an Open Access journal distributed under the Creative Commons Attribution-NonCommercial-NoDerivatives 4.0 International License. To view the details of this license, please visit (https://creativecommons.org/licenses/ by-nc-nd/4.0/).

(C) 2019 The Japanese Society of Internal Medicine

Intern Med 58: 3163-3165, 2019 